\title{
Diferencias clínicas entre preescolares y escolares con apnea obstructiva del sueño
}

\section{Clinical differences between preschoolers and school children with obstructive sleep apnea}

\author{
José Luis Carrillo-Alduenda, ${ }^{1}$ Roberto Agustín Torres-Valerio, ${ }^{1}$ Emma Rosario García-Colín, ${ }^{2}$ María del Rocío \\ Baños-Flores, ${ }^{1}$ Martha Guadalupe Torres-Fraga, ${ }^{1}$ Pablo Brockmann-Veloso ${ }^{3}$
}

\begin{abstract}
Resumen
OBJETIVO: Describir el cuadro clínico, evaluar la repercusión de la edad e identificar los factores asociados con la gravedad de la apnea obstructiva del sueño en un grupo de niños preescolares y escolares.

MATERIALES Y MÉTODOS: Estudio observacional y retrospectivo, efectuado en niños de 3 a 12 años con apnea obstructiva del sueño, atendidos en el Instituto Nacional de Enfermedades Respiratorias Ismael Cosío Villegas, entre el 1 de enero y el 31 de diciembre de 2014. El cuadro clínico se describió con la información contenida en los expedientes; los padres de los niños enviados a la Unidad de Medicina de Sueño respondieron un cuestionario estandarizado de síntomas de sueño y comorbilidades. Para el análisis de la información se utilizó estadística descriptiva. La normalidad de la distribución de los datos se comprobó con la prueba de Kolmogorov Smirnov; las variables dicotómicas se evaluaron con $\chi^{2}$ y las variables continuas con t de Student. RESULTADOS: Se revisaron los expedientes de 108 preescolares y 136 escolares. El $74 \%$ tuvo hipertrofia de amígdalas. Los síntomas más frecuentes fueron: ronquido habitual, respiración oral nocturna y tos crónica $(73,72$ y $70 \%$, respectivamente). Los preescolares tuvieron mayor frecuencia de enfermedad por reflujo gastroesofágico (38 vs $22 \%$ ) y los escolares mayor obesidad (13 vs 39\%) y cardiopatías ( 4 vs $10 \%$, p < 0.05 ). La frecuencia de apnea obstructiva del sueño grave fue mayor en preescolares (76 vs $62 \%, p=0.01)$. En los preescolares, el ronquido, las apneas presenciadas, ahogos nocturnos, vigilancia nocturna por los padres y la hipertrofia de amígdalas se asoció con la gravedad de la enfermedad (RM 3.58, 5.92, 5.37, 5.89, 2.17, respectivamente, $\mathrm{p}<0.05$ ).

CONCLUSIONES: El grupo etario se asocia con diferencias en la manifestación clínica de la apnea obstructiva del sueño, gravedad de la enfermedad y uso del equipo de presión positiva.
\end{abstract}

PALABRAS CLAVE: Apnea obstructiva del sueño; comorbilidades; hipertrofia de amígdala; ronquido; niño.

abstract

OBJECTIVE: To describe the clinical features, assess the impact of age and identify the factors associated with the severity of obstructive sleep apnea in a group of preschool and school children's.

MATERIALS AND METHODS: Observational and retrospective study, conducted in children of 3 to 12 years-old, with obstructive sleep apnea, treated at the National Institute of Respiratory Diseases Ismael Cosío Villegas, between January 1 and December 31, 2014. The clinical was described with the information contained in the records. The parents of the children sent to the Sleep Medicine Unit answered a standardized questionnaire of sleep symptoms and comorbidities. For the analysis of the information descriptive statistics were used. The normality of the data distribution was checked with the Kolmogorov Smirnov test. Dichotomous variables were evaluated with $\chi^{2}$ and continuous variables with Student's t.

RESULTS: 108 preschoolers and 136 schoolchildren were registered. The $74 \%$ had tonsil hypertrophy. The most frequent symptoms were: habitual snoring, nocturnal oral breathing and chronic cough (73, 72 and $70 \%$, respectively). Preschoolers had a higher

\author{
${ }^{1}$ Unidad de Medicina de Sueño. \\ Neumóloga pediatra. \\ Instituto Nacional de Enfermedades \\ Respiratorias Ismael Cosío Villegas, \\ Ciudad de México, México. \\ ${ }^{3}$ Neumólogo pediatra, Centro de \\ Sueño, Escuela de Medicina, Pontificia \\ Universidad Católica de Chile, Santia- \\ go, Chile.
}

Recibido: 4 de abril 2019

Aceptado: 17 de septiembre 2019

Correspondencia José Luis Carrillo Alduenda jlcarrillo14@hotmail.com

Este artículo debe citarse como Carrillo-Alduenda JL, Torres-Valerio RA, García-Colín ER, Baños-Flores MR, Torres-Fraga MG, BrockmannVeloso P. Diferencias clínicas entre preescolares y escolares con apnea obstructiva del sueño. Acta Pediatr Mex. 2019;40(6):318-27. 


\begin{abstract}
frequency of gastroesophageal reflux disease (38\% vs. 22\%) and schoolchildren were more obese $(13 \%$ vs $39 \%)$ and with more heart disease $(4 \%$ vs $10 \%, \mathrm{p}<0.05)$. The frequency of obstructive severe sleep apnea was higher in preschoolers (76 vs. 62\%, $p=0.01$ ). In preschoolers, snoring, witnessed apneas, night drowning, parental night surveillance and tonsil hypertrophy was associated with the severity of the disease (OR $3.58,5.92,5.37,5.89,2.17$, respectively, $\mathrm{p}<0.05)$.

CONCLUSIONS: The age group is associated with differences in the clinical manifestation of obstructive sleep apnea, severity of illness and use of positive pressure equipment. KEYWORDS: Obstructive sleep apnea; Comorbidities; Tonsil hypertrophy; Snore; Boy.
\end{abstract}

\section{ANTECEDENTES}

La apnea obstructiva del sueño es un trastorno caracterizado por el cierre intermitente (parcial o total) de la vía aérea superior al dormir, que altera la ventilación y el patrón normal del sueño y ocasiona hipoxemia e hipercapnia nocturnas. ${ }^{1}$ Su etiología más común en pediatría es la hipertrofia de tejido linfoide faríngeo; $\sin$ embargo, las enfermedades neuromusculares, alteraciones-deformidades craneofaciales y la obesidad representan otras causas conocidas, ${ }^{2}$ ésta última de importancia en nuestro medio, porque supone una epidemia.

La apnea obstructiva del sueño es un padecimiento frecuente, su prevalencia en la población general pediátrica varía de 1-3\% y es más común en el grupo de edad preescolar. Representa un problema creciente de salud pública debido a su elevada morbilidad; ;-6 las complicaciones son frecuentes, incluso pueden ser severas, por lo que la disfunción del patrón del sueño de la apnea obstructiva del sueño puede ocasionar falla de medro, ${ }^{7}$ alteraciones cognitivas y del comportamiento como: retraso del crecimiento, bajo rendimiento escolar, déficit de atenciónhiperactividad, problemas de concentración y memoria, comportamiento agresivo ${ }^{8,9}$ e hipoxia nocturna, que en algunas ocasiones puede generar crisis convulsivas; ${ }^{1}$ incluso, puede acompañarse de complicaciones cardiovasculares, provocadas por la inflamación sistémica y disfunción endotelial, ya sea hipertensión arterial pulmonar, cor pulmonale, hipertensión arterial sistémica, ${ }^{2}$ disfunción autonómica, remodelamiento ventricular y aterogénesis. ${ }^{10}$

En la bibliografía internacional se reporta un cuadro clínico caracterizado por ronquido intenso; los padres de los pacientes refieren escuchar una respiración difícil, con pausas o ahogos nocturnos, y visualizar retracciones de la pared del tórax. El niño puede adoptar posiciones inusuales al dormir (dormir sentado o con el cuello hiperextendido), sufrir diaforesis nocturna y cefalea matutina. Debido a la preocupación, la mayoría de los padres duerme con sus hijos y los estimula para respirar; puede haber somnolencia excesiva diurna, especialmente en escolares y adolescentes; sin embargo, es menos común que en el adulto. ${ }^{1}$

En nuestro medio, el cuadro clínico de los niños con apnea obstructiva del sueño no está bien descrito, incluso se han reportado diferentes factores predisponentes, que varían en sí mismos: edad de manifestación, percepción de los padres y nivel sociocultural de la familia, ${ }^{11}$ que puede retrasar el diagnóstico y tratamiento, y desencadenar complicaciones adicionales.

Con base en lo anterior, el objetivo de este estudio fue: describir el cuadro clínico, las comorbilidades, los hallazgos en el estudio del 
sueño y en la prueba prequirúrgica con equipo de presión positiva, además de evaluar la repercusión de la edad e identificar los factores clínicos asociados con la gravedad de la apnea obstructiva del sueño en un grupo de niños preescolares y escolares.

\section{MATERIALES Y MÉTODOS}

Estudio observacional y retrospectivo, efectuado en el Instituto Nacional de Enfermedades Respiratorias Ismael Cosío Villegas, entre el 1 de enero y el 31 de diciembre de 2014. Criterios de inclusión: pacientes de 3 a 12 años, de uno y otro género, residentes de la Ciudad de México y su área metropolitana, con índice de apneahipopnea $(\mathrm{IAH}) \geq 3$ eventos por hora. ${ }^{12}$ Criterios de exclusión: pacientes con información incompleta, registro de sueño con duración menor de 4 horas, enfermedad neuromuscular, síndrome de Down, labio y paladar hendido, parálisis cerebral, alteraciones craneofaciales, enfermedades congénitas y otros trastornos del sueño diferentes a la apnea obstructiva del sueño.

El cuadro clínico se describió con la información contenida en los expedientes; los padres de los niños enviados a la Unidad de Medicina de sueño respondieron un cuestionario estandarizado de síntomas de sueño y comorbilidades, descrito previamente en niños mexicanos, ${ }^{13}$ que incluye una exploración física. Las preguntas pueden generar respuestas abiertas, dicotómicas (SíNo u Hombre-Mujer), en escala Likert (nunca, rara vez, algunas veces, frecuentemente, por lo general y siempre; en este caso, la pregunta se considera positiva cuando se responde: "por lo general o siempre") o, bien, escala visual análoga (EVA); inicia con las indicaciones para responderlo e incluye tres ejemplos de preguntas contestadas, para posteriormente dividirse en las siguientes siete secciones (el cuestionario completo se presenta en el anexo, las respuestas positivas de la escala Likert están destacadas):
1. Datos generales: incluye la identificación, localización, género y edad del paciente.

2. Antecedentes: investiga los antecedentes perinatales (específicamente prematurez y tabaquismo materno durante el embarazo) y familiares del paciente.

3. Datos del hogar y la familia: se investigan las características generales de la casahabitación, convivencia con animales y tabaquismo pasivo del niño.

4. Hábitos de sueño: es el apartado más importante del cuestionario; incluye horarios de sueño, síntomas al iniciar el sueño o al despertar, siestas, frecuencia de ronquidos e intensidad, apnea o ahogos nocturnos, somnolencia excesiva diurna, cansancio-fatiga e irritabilidad (los tres últimos síntomas se evalúan con la EVA).

5. Salud del niño: se indagan las comorbilidades que padece el paciente.

6. Síntomas respiratorios: la finalidad es obtener información de posibles padecimientos respiratorios, con énfasis en asma y rinitis.

7. Exploración física: se consigna el peso, talla, signos vitales, evaluación de la vía aérea superior y alteraciones mandibulares. Se definió "riesgo" a las siguientes situaciones: apertura bucal clases III o IV de Mallampati; hipertrofia amigdalina (tamaño de las amígdalas grado 3 o 4) ${ }^{14} \mathrm{y}$ alteraciones mandibulares asociadas con micrognatia o retrognatia.

En todos los pacientes se utilizó el equipo Remmers Sleep Recorder ${ }^{\circledR}$ (Sagatech, Calgary, Canadá), que cuenta con los siguientes canales: flujo respiratorio por cánula de presión nasal, ronquido, posición corporal, banda piezoeléctrica torácica para esfuerzo, pulsoximetría y frecuencia cardiaca. Los estudios fueron calificados automáticamente, de acuerdo con el programa de cómputo del fabricante (propor- 
ciona un índice de eventos respiratorios basado en la oximetría, análogo con el índice apneahipopnea [IAH] y su uso en niños mexicanos está descrito previamente). ${ }^{13,15,16}$ Se definió apnea obstructiva del sueño grave al IAH igual o menor de 10 eventos por hora. ${ }^{17}$

Para el procedimiento en nuestro servicio médico, ninguno de los niños recibió tratamiento con esteroides nasales ni antileucotrienos previo al estudio del sueño, y se enviaron al servicio de Otorrinolaringología para tratamiento quirúrgico. Los pacientes en quienes se identificó enfermedad grave y consideró con riesgo quirúrgico elevado recibieron una prueba pre-quirúrgica, con un equipo de presión positiva continua en la vía aérea auto-ajustable (autoCPAP). En todos los casos se utilizó el equipo S7 AutoSet $^{\circledR}$, con humidificador $\operatorname{ResMed}^{\circledR}$ y mascarilla oronasal ajustada a la cara del niño, programado a una presión mínima de $4 \mathrm{~cm} \mathrm{H} \mathrm{H}_{2} \mathrm{O}$ y presión máxima de $15 \mathrm{~cm} \mathrm{H}$ O. Se utilizó el programa ResScan ${ }^{\circledR}$ para obtener la información del equipo, eventos respiratorios residuales, presión y fuga. ${ }^{16}$

Para evaluar la repercusión de la edad, se dividió a los pacientes en grupo 1: preescolares de 3 a 6 años y grupo 2: escolares de 6 a 12 años.

Para el análisis de la información se utilizó estadística descriptiva. Las variables continuas se expresaron en media \pm desviación estándar y las dicotómicas en número (porcentaje); para comparar los horarios de inicio de sueño y despertar, la variable se codificó en número entero a la hora y los minutos se transformaron a su fracción correspondiente. La normalidad de la distribución de los datos se comprobó con la prueba de Kolmogorov Smirnov; las variables dicotómicas se evaluaron con la $\chi^{2}$ y las variables continuas con la t de Student. Para medir la asociación entre la gravedad de la apnea obstructiva del sueño y las variables clínicas se calculó la razón de momios (RM) a través de regresión logística; el valor de p se estableció < 0.05 bimarginal. Se utilizó el programa estadístico STATA 14.

\section{RESULTADOS}

Se revisaron 268 expedientes; sin embargo, 244 (91\%) cumplieron con los criterios de inclusión: 108 preescolares y 136 escolares; $65 \%$ hombres y $45 \%$ mujeres. El motivo más frecuente de exclusión fue llenado incompleto del cuestionario. Los antecedentes familiares más importantes fueron obesidad y alergias ( 45 y $40 \%$ respectivamente); no se encontraron diferencias entre los grupos en esta sección del cuestionario.

Al analizar los hábitos de sueño y la percepción de los padres cuando duermen con sus hijos destacó que los niños van a dormir a las 21:30 $\mathrm{h} \pm 1: 30$, y duermen, en promedio, $10 \mathrm{~h}$. Los escolares despiertan 25 minutos más temprano que los preescolares. Los síntomas más frecuentes fueron: 1) ronquido habitual, 2) respiración oral nocturna y 3 ) tos crónica (73, 72 y $70 \%$, respectivamente); el ronquido fue más intenso en el grupo de escolares $(6 \pm 2$ vs $5.4 \pm 2$ por EVA, respectivamente, $p=0.03$ ). El resto de los síntomas al dormir fueron poco reportados; se encontraron, además, algunas diferencias propias de la edad. Así, los preescolares tienen mayor cantidad de siestas que los escolares; sin embargo, debe considerarse que $16 \%$ de los escolares aún toman una siesta al día.

En el apartado de salud del niño, la obesidad fue más frecuente que la desnutrición (27 vs 8\%); los preescolares tuvieron mayor porcentaje de enfermedad por reflujo gastroesofágico (38 vs $22 \%$, respectivamente, $p=0.00$ ) y los escolares mayor frecuencia de obesidad (13 vs 39, $\mathrm{p}=$ 0.00 ) y cardiopatías (4 vs 10\%, p = 0.04).

A la exploración física se observó buena apertura bucal y la frecuencia de las clases III y IV de Mallampati fue de $41 \%$; se encontró $14 \%$ de 
alteraciones mandibulares y destacó la elevada frecuencia de hipertrofia de amígdalas (74\%), principalmente en preescolares (84 vs 65\%, p $=0.00)$. Las características generales y manifestaciones clínicas de los niños se enlistan en el Cuadro 1.

Cuadro 1. Resumen del cuestionario y comparación del cuadro clínico entre preescolares y escolares (continúa en la siguiente columna)

\begin{tabular}{|c|c|c|c|c|}
\hline Variables & $\begin{array}{c}\text { Total } \\
n= \\
244\end{array}$ & $\begin{array}{c}\text { Preescolares } \\
n=108\end{array}$ & $\begin{array}{c}\text { Escolares } \\
n=136\end{array}$ & $\mathbf{p}^{\&}$ \\
\hline \multicolumn{5}{|l|}{ Datos generales } \\
\hline \multicolumn{5}{|l|}{ Género } \\
\hline Masculino* & $\begin{array}{l}158 \\
(65)\end{array}$ & $63(58)$ & $95(70)$ & 0.06 \\
\hline Femenino* & $86(35)$ & $45(42)$ & $41(30)$ & 0.06 \\
\hline \multicolumn{5}{|l|}{ Antecedentes } \\
\hline Obesidad* & $\begin{array}{l}111 \\
(45)\end{array}$ & $46(43)$ & $65(48)$ & 0.43 \\
\hline Alergias* & $99(41)$ & $47(44)$ & $52(38)$ & 0.47 \\
\hline \multicolumn{5}{|l|}{ Datos del hogar } \\
\hline $\begin{array}{l}\text { Tabaquismo } \\
\text { pasivo* }\end{array}$ & $52(21)$ & $25(23)$ & $27(20)$ & 0.57 \\
\hline Hacinamiento* & $\begin{array}{l}116 \\
(48)\end{array}$ & $51(47)$ & $65(48)$ & 0.85 \\
\hline \multicolumn{5}{|l|}{ Hábitos de sueño } \\
\hline $\begin{array}{l}\text { Horario al } \\
\text { dormir }(\mathrm{h})+\end{array}$ & $\begin{array}{l}21: 30 \\
\pm 1: 42\end{array}$ & $\begin{array}{l}22: 30 \\
\pm 1: 42\end{array}$ & $\begin{array}{l}21: 30 \\
\pm 2\end{array}$ & 0.8 \\
\hline $\begin{array}{l}\text { Dificultad } \\
\text { para iniciar* }\end{array}$ & $62(25)$ & $28(26)$ & $34(25)$ & 0.89 \\
\hline $\begin{array}{l}\text { Horario al } \\
\text { despertar }(\mathrm{h})+\end{array}$ & $\begin{array}{c}7: 30 \\
\pm 1: 36\end{array}$ & $\begin{array}{l}07: 45 \\
\pm 1: 06\end{array}$ & $\begin{array}{l}07: 20 \\
\pm 1: 48\end{array}$ & 0.03 \\
\hline $\begin{array}{l}\text { Dificultad para } \\
\text { despertar* }\end{array}$ & $79(32)$ & $32(30)$ & 47 (35) & 0.32 \\
\hline Siesta* & $55(23)$ & $32(30)$ & $23(17)$ & 0.02 \\
\hline $\begin{array}{l}\text { Ronquido } \\
\text { habitual* }\end{array}$ & $\begin{array}{l}178 \\
(73)\end{array}$ & $85(79)$ & $93(68)$ & 0.12 \\
\hline $\begin{array}{l}\text { Intensidad del } \\
\text { ronquido+ }\end{array}$ & $5.6 \pm 2$ & $6 \pm 2$ & $5.4 \pm 2$ & 0.03 \\
\hline $\begin{array}{l}\text { Apneas } \\
\text { presenciadas* }\end{array}$ & $98(40)$ & $48(44)$ & $50(37)$ & 0.24 \\
\hline $\begin{array}{l}\text { Ahogos } \\
\text { nocturnos* }\end{array}$ & $97(40)$ & $46(43)$ & $51(38)$ & 0.44 \\
\hline
\end{tabular}

Cuadro 1. Resumen del cuestionario y comparación del cuadro clínico entre preescolares y escolares (continúa en la siguiente página)

\begin{tabular}{|c|c|c|c|c|}
\hline Variables & $\begin{array}{c}\text { Total } \\
n=244\end{array}$ & $\begin{array}{c}\text { Preescolares } \\
\mathrm{n}=108\end{array}$ & $\begin{array}{c}\text { Escolares } \\
n=136\end{array}$ & $\mathbf{p}^{\&}$ \\
\hline $\begin{array}{l}\text { Vigilancia } \\
\text { nocturna* }\end{array}$ & $88(36)$ & $40(37)$ & $48(35)$ & 0.77 \\
\hline $\begin{array}{l}\text { Somnolencia } \\
\text { excesiva } \\
\text { Diurna+ }\end{array}$ & $\begin{array}{l}3.7 \pm \\
2.6\end{array}$ & $4 \pm 2.5$ & $3.5 \pm 2.7$ & 0.15 \\
\hline Cansancio+ & $\begin{array}{c}3.8 \\
\pm 2.4\end{array}$ & $3.6 \pm 2.4$ & $4 \pm 2.4$ & 0.3 \\
\hline Irritabilidad+ & $\begin{array}{c}4.4 \\
\pm 2.8\end{array}$ & $4.7 \pm 2.7$ & $4.2 \pm 2.8$ & 0.25 \\
\hline $\begin{array}{l}\text { Somnolencia } \\
\text { en clase* }\end{array}$ & $44(18)$ & $18(17)$ & $26(19)$ & 0.68 \\
\hline $\begin{array}{l}\text { Respiración } \\
\text { oral diurna* }\end{array}$ & $\begin{array}{l}140 \\
(57)\end{array}$ & $65(60)$ & $75(55)$ & 0.55 \\
\hline $\begin{array}{l}\text { Respiración } \\
\text { oral noctur- } \\
\text { na* }\end{array}$ & $\begin{array}{l}176 \\
(72)\end{array}$ & $79(73)$ & $97(71)$ & 0.89 \\
\hline \multicolumn{5}{|l|}{ Salud del niño } \\
\hline Talla baja* & $69(28)$ & $35(32)$ & $34(25)$ & 0.16 \\
\hline Desnutrición* & $20(8)$ & 9 (8) & $11(8)$ & 0.94 \\
\hline Obesidad* & $67(27)$ & $14(13)$ & $53(40)$ & 0.00 \\
\hline ERGE* & $71(29)$ & $41(38)$ & $30(22)$ & 0.00 \\
\hline Cardiopatía* & $18(7)$ & $4(4)$ & $14(10)$ & 0.04 \\
\hline \multicolumn{5}{|c|}{ Síntomas respiratorios } \\
\hline Tos crónica* & $\begin{array}{l}170 \\
(70)\end{array}$ & $79(73)$ & $91(67)$ & 0.31 \\
\hline Asma* & $65(27)$ & $24(22)$ & $41(30)$ & 0.14 \\
\hline $\begin{array}{l}\text { Obstrucción } \\
\text { nasal* }\end{array}$ & $\begin{array}{l}119 \\
(49)\end{array}$ & $57(52)$ & $62(46)$ & 0.37 \\
\hline Rinorrea* & $50(20)$ & $25(23)$ & $25(18)$ & 0.42 \\
\hline \multicolumn{5}{|l|}{ Exploración física } \\
\hline Peso (kg)+ & $\begin{array}{c}27.4 \\
\pm 14.6\end{array}$ & $18 \pm 5.3$ & $\begin{array}{c}35 \\
+15.2\end{array}$ & $\mathrm{NE}$ \\
\hline Talla $(\mathrm{cm})+$ & $\begin{array}{c}120 \\
\pm 17.6\end{array}$ & $106 \pm 9.7$ & $129 \pm 21$ & $\mathrm{NE}$ \\
\hline $\mathrm{IMC}\left(\mathrm{kg} / \mathrm{m}^{2}\right)+$ & $\begin{array}{r}17.8 \\
\pm 4.6\end{array}$ & $15.7 \pm 2.7$ & $\begin{array}{l}29.5 \\
\pm 3.7\end{array}$ & $\mathrm{NE}$ \\
\hline Score Z IMC & $\begin{array}{l}0.18 \\
\pm 1.8\end{array}$ & $-0.33 \pm 1.96$ & $\begin{array}{c}0.6 \\
+1.59\end{array}$ & 0.00 \\
\hline $\mathrm{SpO}_{2}(\%)+$ & $\begin{array}{r}95.6 \\
\pm 1.8\end{array}$ & $95.4 \pm 19$ & $\begin{array}{r}95.7 \\
\pm 1.7\end{array}$ & 0.19 \\
\hline
\end{tabular}


Cuadro 1. Resumen del cuestionario y comparación del cuadro clínico entre preescolares y escolares (continuación)

\begin{tabular}{l|cc|c|c|}
\hline Variables & $\begin{array}{c}\text { Total } \\
\mathbf{n = 2 4 4}\end{array}$ & $\begin{array}{c}\text { Preescolares } \\
\mathbf{n = 1 0 8}\end{array}$ & $\begin{array}{c}\text { Escolares } \\
\mathbf{n = 1 3 6}\end{array}$ & $\mathbf{p}^{\&}$ \\
\hline $\begin{array}{l}\text { Mallampati } \\
\text { III-IV* }\end{array}$ & $\begin{array}{r}100 \\
(41)\end{array}$ & $49(45)$ & $51(38)$ & 0.39 \\
$\begin{array}{l}\text { Hipertrofía } \\
\text { amigdalina* }\end{array}$ & $\begin{array}{l}180 \\
(74)\end{array}$ & $91(84)$ & $89(65)$ & 0.00 \\
$\begin{array}{l}\text { Alteraciones } \\
\text { mandibulares* }\end{array}$ & $34(14)$ & $14(13)$ & $20(15)$ & 0.55 \\
& & & &
\end{tabular}

cm: centímetros; ERGE: enfermedad por reflujo gastroesofágico; NE: no evaluado; $\mathrm{SpO}_{2}$ saturación de oxígeno.

La hipertrofia amigdalina se definió como el tamaño de amígdalas grado 3 o 4 .

Alteraciones mandibulares se definió como la presencia de micrognatia o retrognatia.

* Variable resumida en $\mathrm{n}(\%)$, todos los porcentajes fueron redondeados a número entero más próximo.

+ Variable resumida en media \pm desviación estándar.

\& Comparación entre preescolares vs escolares.

Al comparar los hallazgos en el estudio del sueño se observó que los parámetros respiratorios fueron más graves en los niños preescolares versus escolares; por tanto, tuvieron un IAH mayor (26 \pm 24 vs $\left.19 \pm 22.3 \mathrm{~h}^{-1}, \mathrm{p}=0.01\right), \mathrm{SpO}_{2}$ nocturna más baja $(73 \% \pm 11$ vs $78.9 \% \pm 9, p=0.00)$ y elevada prevalencia de casos graves (76 vs $62 \%$, $p=0.01)$. Cuadro 2

Por indicación del médico tratante, a 58 pacientes (34 preescolares y 24 escolares) se les realizó una prueba prequirúrgica con un equipo de presión positiva continua en la vía aérea autoajustable (autoCPAP, siglas en Inglés) durante una semana, donde se observó menor apego al dispositivo en el grupo de niños preescolares, además de menos horas promedio de uso por noche $(4.9 \mathrm{~h} \pm 0.5$ vs $6.7 \mathrm{~h} \pm 0.4, \mathrm{p}=0.00) \mathrm{y}$ menor porcentaje de días de uso, es decir, más $\mathrm{de}<4 \mathrm{~h}(61.7 \% \pm 37.8$ vs $88.7 \% \pm 18, \mathrm{p}=0.00)$. En esta prueba terapéutica el equipo fue efectivo para eliminar los eventos respiratorios. El índice de apnea-hipopnea disminuyó de $22.5 \pm 23.5$ a $7.2 \pm 4.4, p=0.00$. El percentil 95 de presión, que representa la presión terapéutica fue de 10.4
Cuadro 2. Hallazgos en el estudio del sueño

\begin{tabular}{|c|c|c|c|c|}
\hline Variables & $\begin{array}{c}\text { Total } \\
n=244\end{array}$ & $\begin{array}{c}\text { Preescolares } \\
\mathbf{n}=\mathbf{1 0 8}\end{array}$ & $\begin{array}{l}\text { Escolares } n \\
\quad=136\end{array}$ & $p^{\&}$ \\
\hline $\mathrm{IAH}+$ & $\begin{array}{c}22.5 \\
\pm 23.5\end{array}$ & $26 \pm 24$ & $\begin{array}{c}19.2 \\
\pm 22.3\end{array}$ & 0.01 \\
\hline $\begin{array}{l}\mathrm{SpO}_{2} \\
\text { promedio+ }\end{array}$ & $91.5 \pm 3.7$ & $91 \pm 4$ & $91.7 \pm 3.3$ & 0.19 \\
\hline TC90\%+ & $16 \pm 25.6$ & $19.5 \pm 26$ & $13.2 \pm 24$ & 0.05 \\
\hline $\mathrm{SpO}_{2} \min +$ & $\begin{array}{c}75.9 \\
\pm 10.5\end{array}$ & $73 \pm 11$ & $78.9 \pm 9$ & 0.00 \\
\hline $\begin{array}{l}\text { AOS } \\
\text { grave }\end{array}$ & 113 (46) & $83(77)$ & $84(62)$ & 0.01 \\
\hline
\end{tabular}

AOS: apnea obstructiva del sueño; IAH: índice de apneahipopnea; min: mínimo; SpO2: saturación de oxígeno; TC90\%: tiempo de saturación $<90 \%$.

Se definió AOS grave al índice de $\mathrm{IAH} \geq 10$ eventos por hora. * Variable resumida en $\mathrm{n}(\%)$, todos los porcentajes fueron redondeados a su número entero más próximo.

+ Variable resumida en media \pm desviación estándar. \& Comparación entre preescolares vs escolares.

$\pm 2 \mathrm{~cm} \mathrm{H}_{2} \mathrm{O}$, sin diferencia estadística entre los grupos. Cuadro 3

Algunas variables clínicas se asociaron con mayor riesgo de apnea obstructiva del sueño grave, especialmente en los preescolares; así, en los niños de 3 a 6 años, el ronquido habitual (RM 3.58 IC95\%: 1.32-9.71), la apnea presenciada (RM 5.92 IC95\%: 1.87-18.7), los ahogos nocturnos (RM 5.37, IC95\%: 1.69-17.02), la vigilancia nocturna por los padres (RM 5.89 IC95\%: 1.63-21.2) y la hipertrofia amigdalina (OR 2.17 IC95\%: 1.21-3.91) incrementaron el riesgo de apnea obstructiva del sueño grave, mientras que en el grupo de 6 a 12 años solo los ahogos nocturnos mostraron una asociación estadísticamente significativa (RM 5.37 IC95\%: 2.25-12.8). Las comparaciones por género no mostraron diferencias estadísticas.

\section{DISCUSIÓN}

Del cuadro clínico de niños de 3 a 12 años con apnea obstructiva del sueño, residentes de la 
Cuadro 3. Prueba prequirúrgica con equipo de presión positiva

\begin{tabular}{|l|c|c|c|c|}
\hline Variables & Total $\mathbf{n}=\mathbf{5 8}$ & Preescolares $\mathbf{n}=\mathbf{3 4}$ & Escolares $\mathbf{n}=\mathbf{2 4}$ & $\mathbf{p}^{\mathbf{4}}$ \\
\hline Días de uso $>4 \mathrm{~h}(\%)$ & $73 \pm 33.8$ & $61.7 \pm 37.8$ & $88.7 \pm 18$ & 0.00 \\
\hline Horas de uso $(\mathrm{h}$ [promedio]) & $5.7 \pm 2.7$ & $4.9 \pm 0.5$ & $6.7 \pm 0.4$ & 0.00 \\
\hline IAH residual $\left(\mathrm{h}^{-1}\right)$ & $7.2 \pm 4.4$ & $7.5 \pm 0.8$ & $6.7 \pm 0.6$ & 0.5 \\
\hline IA residual $\left(\mathrm{h}^{-1}\right)$ & $1.4 \pm 1.9$ & $1.1 \pm 0.2$ & $1.8 \pm 0.4$ & 0.11 \\
\hline IH residual $\left(\mathrm{h}^{-1}\right)$ & $5.8 \pm 4.1$ & $6.4 \pm 0.7$ & $4.8 \pm 0.6$ & 0.15 \\
\hline P95 presión $\left(\mathrm{cmH}_{2} \mathrm{O}\right)$ & $10.4 \pm 2$ & $10.3 \pm 0.4$ & $10.4 \pm 0.3$ & 0.9 \\
\hline P95 fuga $(\mathrm{L} / \mathrm{seg})$ & $1.2 \pm 3.9$ & $1 \pm 0.4$ & $1.6 \pm 1$ & 0.4 \\
\hline
\end{tabular}

$\mathrm{CmH}_{2} \mathrm{O}=$ centímetros de agua, $\mathrm{h}=$ horas, $\mathrm{h}^{-1}=$ eventos por hora, $\mathrm{IA}=$ índice de apnea, $\mathrm{IAH}=$ índice de apnea hipopnea, $\mathrm{IH}=$ índice de hipopnea, L/seg= litros por segundo, P95= percentil 95.

Todas las variables se resumieron como media \pm desviación estándar.

\& comparación entre preescolares vs escolares.

Ciudad de México, destaca que se acuestan a las 21:30 h y duermen, en promedio, $10 \mathrm{~h}$. Esta práctica se encuentra en el límite inferior de las horas de sueño recomendadas por la Academia Americana de Medicina de Sueño como hábito saludable óptimo, pues un preescolar debería dormir entre 10 y 13 horas al día (incluida siesta) y un escolar entre 9 y 12 horas al día (a esta edad no se recomienda la siesta). ${ }^{18}$ La disminución de las horas de sueño en niños mexicanos se ha descrito con anterioridad; por ejemplo, Arana-Lechuga y sus colaboradore ${ }^{19}$ reportaron un tiempo total de sueño de $8.8 \pm 1.6 \mathrm{~h}$, en un grupo de 355 niños de 8 a 14, y la información se obtuvo a través de un cuestionario autoaplicable a los padres; sin embargo, una buena parte de la muestra estuvo conformada por adolescentes, quienes duermen menos que los preescolares y escolares, lo que podría explicar las diferencias con nuestros pacientes.

Los niños con apnea obstructiva del sueño pueden manifestar diversos síntomas diurnos y nocturnos; sin embargo, el ronquido es el referido con mayor frecuencia en las revisiones y series de casos. ${ }^{20}$ Carroll y sus colaboradores, en su estudio con 35 pacientes de $4.3 \pm 2.4$ años (mínima de 5 meses y máxima de 14 años) con apnea obstructiva del sueño, reportaron ronquido intenso en $91 \%$ de los casos, ronquido casi todas las noches en $97 \%$, apnea en $74 \%$, respiración nocturna difícil (ahogos nocturnos) en $89 \%$, cianosis nocturna en $6 \%$, necesidad de movilizar al niño para estimular su respiración en $60 \%$, vigilancia nocturna por parte de los padres en $91 \%$ y respiración oral en $85 \% .{ }^{21}$ Estos datos coinciden con los nuestros en la alta frecuencia de ronquido; no obstante, destaca el bajo reporte de apneas presenciadas, ahogos nocturnos y vigilancia nocturna en nuestros pacientes, que puede explicarse por las diferencias raciales $y$ culturales entre las poblaciones y por la forma de recolección de los datos, pues en el estudio de Carroll y su grupo obtuvieron la información del expediente y, quizá, la pregunta fue directamente elaborada a los padres por un médico, mientras que en nuestro caso la obtuvimos de un cuestionario auto-aplicado.

La hipertrofia de amígdalas y su asociación con la gravedad de la apnea obstructiva del sueño aún se discute. Una revisión sistemática que incluyó 20 artículos, ${ }^{22}$ con 3353 pacientes de 6.7 años (intervalo de 2.7 a 11.7), cuyo objetivo fue 
determinar si el tamaño de las amígdalas se relaciona con el índice de apnea-hipopnea, reportó que Nolan y colaboradores no pudieron demostrar la relación entre el tamaño de las amígdalas y la gravedad de la apnea obstructiva del sueño. En nuestros pacientes encontramos relación entre la hipertrofia de amígdalas y la gravedad de la enfermedad, pero solo en los niños preescolares. Este hallazgo tiene plausibilidad biológica entre los 3 y 5 años de edad, pues los tejidos blandos crecen más rápido que la cavidad faríngea, con subsiguiente disminución del volumen de la vía aérea superior en ese periodo. ${ }^{23}$ Arens y su grupo ${ }^{24}$ encontraron menor volumen faríngeo en niños con apnea obstructiva del sueño que en niños sanos, ${ }^{24}$ por lo que en preescolares el reducido crecimiento de los huesos de la cara, secundario a la edad, parece ser un factor de riesgo tan importante como la hipertrofia de tejido linfoide per se. ${ }^{25}$

La obesidad es un factor de riesgo conocido para apnea obstructiva del sueño en niños y adultos; así, Redline y sus colaboradores informaron un riesgo de 4.49 (IC95\%: 1.58 a 13.33) para apnea asociada con obesidad en un grupo de 399 niños de 2 a 18 años. ${ }^{26}$ Por su parte, Kholer y sus colaboradores, ${ }^{27}$ en un grupo de 190 niños caucásicos, encontraron una asociación discreta pero significativa entre obesidad y gravedad de la apnea obstructiva del sueño; sin embargo, la mayoría de los niños con esta alteración no eran obesos. ${ }^{11}$ Este último hallazgo se mantuvo en nuestros pacientes, no obstante, la prevalencia de obesidad fue mayor a la reportada para una zona urbana mexicana: $6.1 \%$ para preescolares y $16.5 \%$ para escolares versus 13 y $39 \%$ en la población estudiada. ${ }^{28}$

En nuestro análisis no demostramos la relación entre obesidad y gravedad de la apnea obstructiva del sueño; sin embargo, la obesidad como factor de riesgo para apnea obstructiva del sueño grave parece depender de la raza. Weinstock y sus coautores, quienes evaluaron 464 niños de 5 y 9.9 años, concluyeron que la obesidad, como mecanismo generador de apnea obstructiva del sueño grave, tiene relevancia solo en niños caucásicos, ${ }^{29}$ si bien este estudio describió que $40 \%$ de sus pacientes tenía descendencia hispana, este dato no se incluyó en el análisis por raza.

La gravedad de la apnea obstructiva del sueño y peores índices de oxigenación entre los preescolares puede explicarse por el menor volumen faríngeo secundario a la relación entre el tamaño de las amígdalas-adenoides y el diámetro de la cavidad (vide supra), ${ }^{25}$ esto puede repercutir negativamente en el volumen corriente y, con ello, en la oxigenación; por tanto, si este hallazgo se asocia con menor número de horas de uso del dispositivo de presión positiva en la prueba prequirúrgica, coloca a los niños preescolares con apnea obstructiva del sueño en una situación de desventaja y vulnerabilidad, que podría generar complicaciones irreversibles a largo plazo.

Entre las limitaciones de este estudio, el cuestionario puede reflejar la opinión subjetiva del cuidador (generalmente la madre) y verse modificada por la edad del niño; además, no se estableció el diagnóstico con polisomnografía, estudio de referencia para el diagnóstico de apnea obstructiva del sueño; sin embargo, el monitor simplificado y la oximetría son métodos útiles, válidos y cada vez más utilizados para diagnosticar la alteración en niños, especialmente en instituciones con recursos limitados. ${ }^{30}$ Para solventar esta cuestión se utilizó IAH $\geq 3$ para definir la apnea obstructiva del sueño ${ }^{12}$ (mayor al $\mathrm{IAH} \geq 1$ sugerido internacionalmente), ${ }^{1}$ este punto de corte está validado y aumentó la especificidad del diagnóstico y, con ello, su validez interna. Otra limitante fue la población de estudio: niños con apnea obstructiva del sueño que recibieron atención en una unidad de sueño de 
tercer nivel de atención, lo que limita la validez externa en este grupo de pacientes.

Las ventajas de este estudio consisten en que representan el quehacer clínico cotidiano de una unidad de sueño de referencia nacional, que utiliza técnicas clínicas fácilmente reproducibles y proporciona información original y útil, de acuerdo con las diferencias raciales propias de la patología, razón por la que la Academia Americana de Medicina del Sueño recomienda investigaciones epidemiológicas locales. ${ }^{31}$

\section{CONCLUSIONES}

Los hallazgos clínicos más frecuentes en los niños con apnea obstructiva del sueño, residentes de la Ciudad de México, son: ronquido habitual, respiración oral nocturna, tos crónica e hipertrofia de amígdalas; el grupo etario se asocia con diferencias en la manifestación clínica, gravedad de la enfermedad y uso prequirúrgico del equipo de presión positiva.

\section{REFERENCIAS}

1. American Academy of Sleep Medicine. International classification of sleep disorders. $3^{\text {rd }}$ ed. Darien, IL: American Academy of Sleep Medicine, 2014.

2. Torre-Bouscoulet L, et al. Fisiopatología del síndrome de apneas-hipopneas durante el sueño en niños. Neumol Cir Torax 2010;69(1):31-8.

3. Ng DK, et al. Prevalence of sleep problems in Hong Kong primary school children: a community-based telephone survey. Chest 2005;128(3):1315-23. DOI: 10.1378/ chest.128.3.1315

4. Rosen $\mathrm{CL}$, et al. Prevalence and risk factors for sleep-disordered breathing in 8- to 11-year-old children: association with race and prematurity. J Pediatr 2003;142(4):383-9. DOI: $10.1067 / \mathrm{mpd} .2003 .28$

5. Spruyt K, et al. Odds, prevalence and predictors of sleep problems in school-age normal children. J Sleep Res 2005;14(2):163-76. DOI: 10.1111/j.13652869.2005.00458.x

6. Brunetti $L$, et al. Prevalence of obstructive sleep apnea syndrome in a cohort of 1,207 children of southern Italy. Chest 2001;120(6):1930-5. DOI: 10.1378/chest.120.6.1930
7. Goodwin JL, et al. Symptoms related to sleep-disordered breathing in white and Hispanic children: the Tucson Children's Assessment of Sleep Apnea Study. Chest 2003;124(1):196-203. DOI: 10.1378/chest.124.1.196

8. Gozal D, et al. Neurocognitive and behavioral morbidity in children with sleep disorders. Curr Opin Pulm Med 2007;13(6):505-9. DOI: 10.1097/MCP.0b013e3282ef6880

9. Esposito $\mathrm{M}$, et al. Executive dysfunction in children affected by obstructive sleep apnea syndrome: an observational study. Neuropsychiatr Dis Treat 2013;9:1087-94. DOI: 10.2147/NDT.S47287

10. Bhattacharjee R, et al. Cardiovascular complications of obstructive sleep apnea syndrome: evidence from children. Prog Cardiovasc Dis 2009;51(5):416-33. DOI: 10.1016/j. pcad.2008.03.002

11. Carroll JL. Obstructive Sleep-disordered breathing in children: new controversies, new directions. Clin Chest Med 2003;24(2):261-82.

12. Alonso-Álvarez ML, et al. Consensus document on sleep apnea-hypopnea síndrome in children (full version). Arch Bronconeumol 2011;47(Supl 5):2-18. DOI: 10.1016/S03002896(11)70026-6

13. Vázquez JC, et al. Clinical Predictors of Sleep Disordered Breathing in Children at Moderate Altitude. Arch Med Res 2004;35(6):525-31. DOI: 10.1016/j.arcmed.2004.11.012

14. Kumar HV, et al. Mallampati score and pediatric sleep apnea. J Clin Sleep Med 2014;10(9):985-90. DOI: 10.5664/jcsm.4032

15. Kirk VG, et al. Comparison of home oximetry monitoring with laboratory polysomnography in children. Chest 2003;124(5):1702-8. DOI: 10.1378/chest.124.5.1702

16. Castorena-Maldonado $\mathrm{A}$, et al. Preoperative continuous positive airway pressure compliance in children with obstructive Sleep apnea syndrome: assessed by a simplified approach. Int J Pediatr Otorhinolaryngol 2008;72(12):17951800. DOI: 10.1016/j.ijporl.2008.08.016

17. Dehlink $E$, et al. Update on paediatric obstructive sleep apnoea. J Thorac Dis 2016;8(2):224-35. DOI: 10.3978/j. issn.2072-1439.2015.12.04

18. Paruthi S, et al. Consensus Statement of the American Academy of Sleep Medicine on the Recommended Amount of Sleep for Healthy Children: Methodology and Discussion. J Clin Sleep Med 2016;12(11):1549-61. DOI: 10.5664/ jcsm.6288

19. Arana-Lechuga DY, et al. Reducción del número de horas de Sueño en niños mexicanos y su impacto en el sobrepeso. An Méd Asoc Med Hosp ABC 2016;61(2):117-22. https://www. medigraphic.com/pdfs/abc/bc-2016/bc162g.pdf

20. Sterni LM, et al. Obstructive sleep apnea in children An Update. Pediatr Clin N Am 2003;50(2):427-43. DOI: 10.1016/ s0031-3955(03)00037-3

21. Carroll JL, et al. Inability of clinical history to distinguish primary snoring from obstructive sleep apnea syndrome in children. Chest 1995;108(3):610-8. DOI: 10.1378/ chest.108.3.610 
22. Nolan J, et al. Systematic review of pediatric tonsil size and polysomnogram-measured obstructive sleep apnea severity. Otolaryngol Head Neck Surg 2011;144(6):844-50. DOI: 10.1177/0194599811400683

23. Jeans WD, et al. A longitudinal study of the growth of the nasopharynx and its contents in normal children. $\mathrm{Br}$ J Radiol 1981;54(638):117-21. DOI: 10.1259/0007-128554-638-117

24. Arens $\mathrm{R}$, et al. Upper airway size analysis by magnetic resonance imaging of children with obstructive sleep apnea syndrome. Am J Respir Crit Care Med 2003;167(1):65-70. DOI: 10.1164/rccm.200206-6130C

25. Papaioannou G, et al. Age-dependent changes in the size of adenotonsillar tissue in childhood: implications for sleepdisordered breathing. J Pediatr 2013;162:269-74. DOI: 10.1016/j.jpeds.2012.07.041

26. Redline $\mathrm{S}$, et al. Risk factors for sleep-disordered breathing in children. Associations with obesity, race, and respiratory problems. Am J Respir Crit Care Med 1999;159(5Pt1):152732. DOI: $10.1164 /$ ajrccm.159.5.9809079

27. Kohler $\mathrm{M}$, et al. Obesity and risk of sleep related upper airway obstruction in Caucasian children. J Clin Sleep Med. 2008;4(2):129-36.

28. Shamah-Levy T, et al. Sobrepeso y obesidad en niños y adolescentes en México, actualización de la Encuesta Nacional de Salud y Nutrición de Medio Camino 2016. Salud Publica Mex 2018;60(3):244-53. http://saludpublica.mx/index.php/ spm/article/view/8815

29. Weinstock TG, et al. Predictors of obstructive sleep apnea severity in adenotonsillectomy candidates. Sleep 2014;37(2):261-69. DOI: 10.5665/sleep.3394

30. Hornero R, et al. Nocturnal Oximetry-based Evaluation of Habitually Snoring Children. Am J Respir Crit Care Med 2017;196(12):1591-98. DOI: 10.1164/rccm.201705-09300C

31. Marcus $\mathrm{CL}$, et al. Diagnosis and management of childhood obstructive sleep apnea syndrome. Pediatrics 2012;130(3):e714-55. DOI: 10.1542/peds.2012-1672 